\title{
Investigation on the Crack Initiation of V-Shaped Notch Tip in Precision Cropping
}

\author{
Lijun Zhang, ${ }^{1}$ Shengdun Zhao, ${ }^{2}$ and Zhenwei Wang ${ }^{2}$ \\ ${ }^{1}$ College of Electromechanical Engineering, China University of Petroleum, Qingdao 266580, China \\ ${ }^{2}$ School of Mechanical Engineering, Xian Jiaotong University, Xian 710049, China \\ Correspondence should be addressed to Lijun Zhang; zlj-2@163.com
}

Received 12 March 2014; Accepted 23 July 2014; Published 21 August 2014

Academic Editor: Jun Liu

Copyright (c) 2014 Lijun Zhang et al. This is an open access article distributed under the Creative Commons Attribution License, which permits unrestricted use, distribution, and reproduction in any medium, provided the original work is properly cited.

\begin{abstract}
The crack initiation of $\mathrm{V}$-shaped notch tip has a very important influence on the cross-section quality and the cropping time for every segment of metal bar in course of low stress precision cropping. By the finite element method, the influence of machining precision of $\mathrm{V}$-shaped notch bottom corner on the crack initiation location is analyzed and it is pointed out that the crack initiation point locates in the place at the maximal equivalent stress change rate on V-shaped notch surface. The judgment criterion of the crack initiation direction is presented and the corresponding crack initiation angle can be calculated by means of the displacement extrapolation method. The factual crack initiation angle of the metal bar has been measured by using the microscopic measurement system. The formula of the crack initiation life of V-shaped notch tip is built, which mainly includes the stress concentration factor of V-shaped notch, the tensile properties of metal material, and the cyclic loading conditions. The experimental results show that the obtained theoretical analyses about the crack initiation location, the crack initiation direction, and the crack initiation time in this paper are correct. It is also shown that the crack initiation time accounts for about $80 \%$ of the cropping time for every segment of the metal bar.
\end{abstract}

\section{Introduction}

In recent years the precision cropping of metal bar in the manufacturing has been paid more and more attention. The so-called cropping refers to the process in which the metal bar is separated into the blank with the desired size and shape. In the machinery manufacturing industry, the cropping course of metal bar is the first process and is widely used in industrial production, such as chain pins, micromotor shafts, and combustion engineer appurtenances. At the present time, the common cropping method in industry is to shear bar directly with the common die, and this process is plagued with a big shearing force, a bad cross-section, a short lifetime die, and noise. Therefore, a new type of low stress cropping method was proposed and gradually used in industrial practice [1-3]. In the low stress cropping, the annular $\mathrm{V}$-shaped notch on the surface circumference of metal bar is processed in advance and it skillfully utilizes the crack technology and the fatigue loading to make the crack checking and propagating along the prefabricated V-shape notch section for shearing the bar.
However, the annular V-shaped notch is generally processed by turning tool with a corner radius, and the bottom of Vshaped notch is an arc, not a crack. Based on it, the initial cropping force is still large and the cropping time for every segment of metal bar is also long in present low stress cropping method. In addition, according to the different stress modes in tip of $\mathrm{V}$-shaped notch, the crack initiation behavior is also different. To obtain the high-quality cropping cross-section, the initiation direction of the crack in tip of Vshaped notch should be perpendicular to the axial cord of the metal bar along the V-shaped notch section. Therefore, it is very important to study the relationship between the stress state of V-shaped notch tip and the crack initiation location and the crack initiation direction in detail, which is very helpful to improve the cropping cross-section quality.

The crack initiation mainly includes three important aspects, namely, the initiation location, the initiation direction, and the initiation time. It is pointed out that the crack initiation time is far more than the summation of the crack extension time and the transient fracture time [4]. Moreover, 


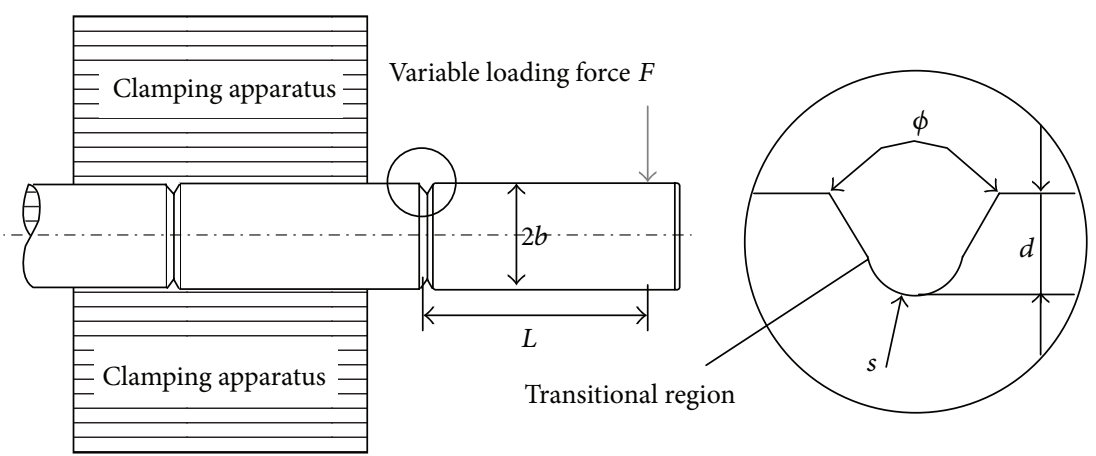

FIGURE 1: Schematic diagram of bar loading and V-shaped notch.

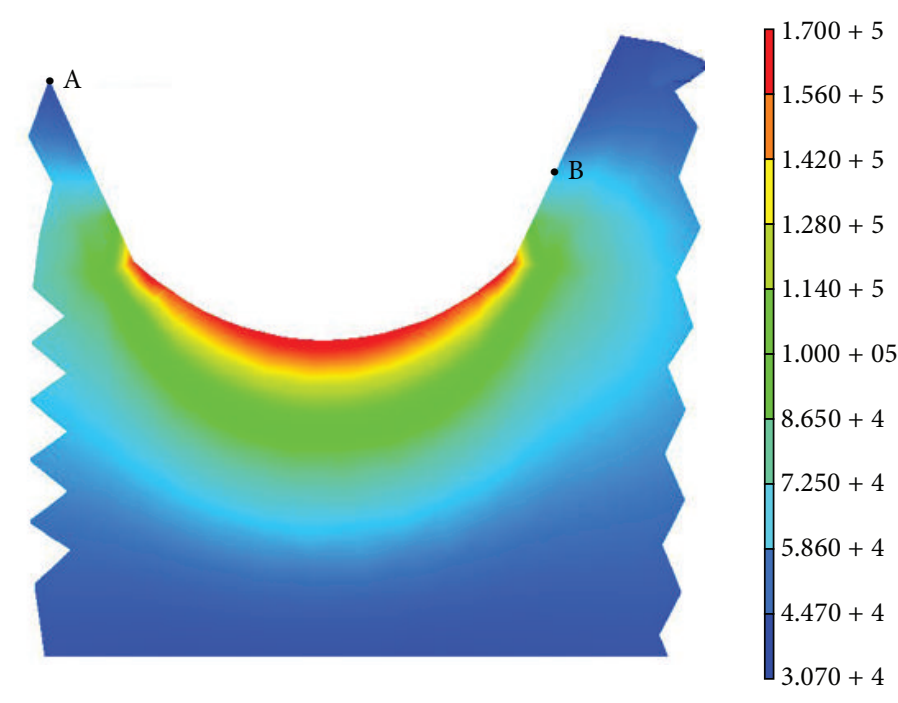

Figure 2: The equivalent stress nephogram near V-shaped notch surface.

the crack initiation time is difficult to compute. Therefore, the theoretical and experimental studies presented in this paper aim to analyze the influences of main factors on the crack initiation location, the crack initiation direction, and the crack initiation time in order to get the desired methods of improving the cropping cross-section quality and calculating the crack initiation life.

\section{The Analysis of Crack Initiation Location Near V-Shaped Notch Tip}

2.1. Influence of Machining Precision of V-Shaped Notch Bottom Corner on the Crack Initiation Location. In course of the low stress cropping, the bar loading and V-shaped notch are schematically shown in Figure 1. As shown in Figure 1, Vshaped notch part consists of the bottom corner and two sides of straight surface. The crack initiation location near $\mathrm{V}$-shaped notch tip is studied by finite element method. The diameter $2 b$ of metal bar is $15 \mathrm{~mm}$, the length $L$ of every segment of bar is $40 \mathrm{~mm}$, the flare angle $\phi$ of V-shaped notch is $60^{\circ}$, the radius $s$ at the notch bottom is $0.3 \mathrm{~mm}$, the notch depth $d$ is $2 \mathrm{~mm}$, and the displacement load is $0.5 \mathrm{~mm}$.
Based on it, the nephogram of equivalent stress of V-shaped notch surface is shown in Figure 2 by ANSYS software. The equivalent stress distribution of the selected points at two sides of the surface near V-shaped notch bottom is obtained in Figure 3. As shown in Figures 2 and 3, the main stress area is located at the bottom of $\mathrm{V}$-shaped notch for 45 steel bars. The stress change of $\mathrm{V}$-shaped notch bottom is not very severe. Because of geometric discontinuities of $\mathrm{V}$-shaped notch surface, the stress mutation occurs in the transitional region between $\mathrm{V}$-shaped notch bottom corner and two sides of straight surface of $\mathrm{V}$-shaped notch. The maximum equivalent stress at the left side of $\mathrm{V}$-shaped notch is about $168 \mathrm{MPa}$, and the maximum equivalent stress at the right side is about $166 \mathrm{MPa}$. Therefore, the equivalent stress at the left side is slightly larger than the one at the right side.

Microcrack is often produced on the surface of the metal material firstly, gradually extends to the interior material, and finally causes the material fracture. As shown in Figure 3, there are three peak values of equivalent stress on $\mathrm{V}$-shaped notch surface and these values have little difference. Therefore, the crack initiation location may appear in any one of the three places. Therefore, it is very difficult to judge the crack initiation location by only relying on the value of maximum 


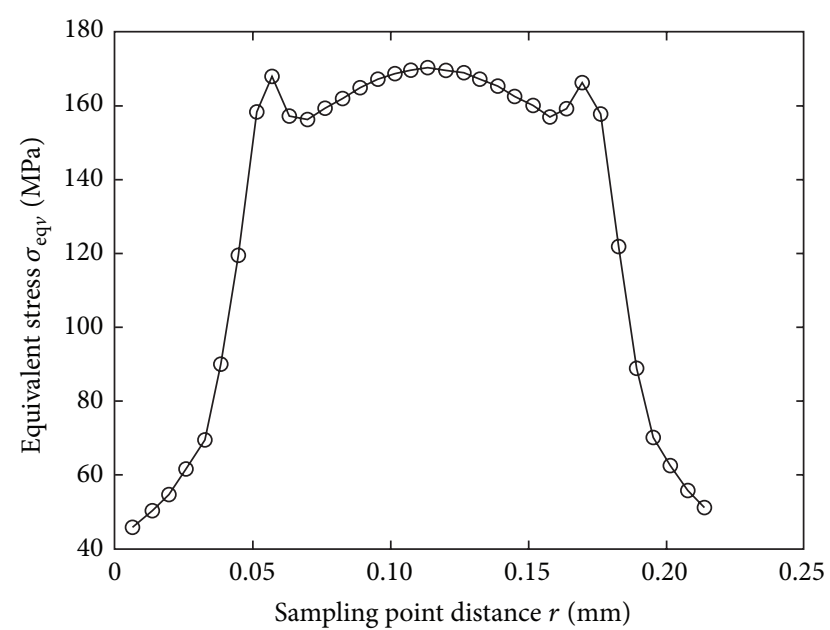

FIGURE 3: The equivalent stress distribution of selected points on two sides of the surface of $\mathrm{V}$-shaped notch.

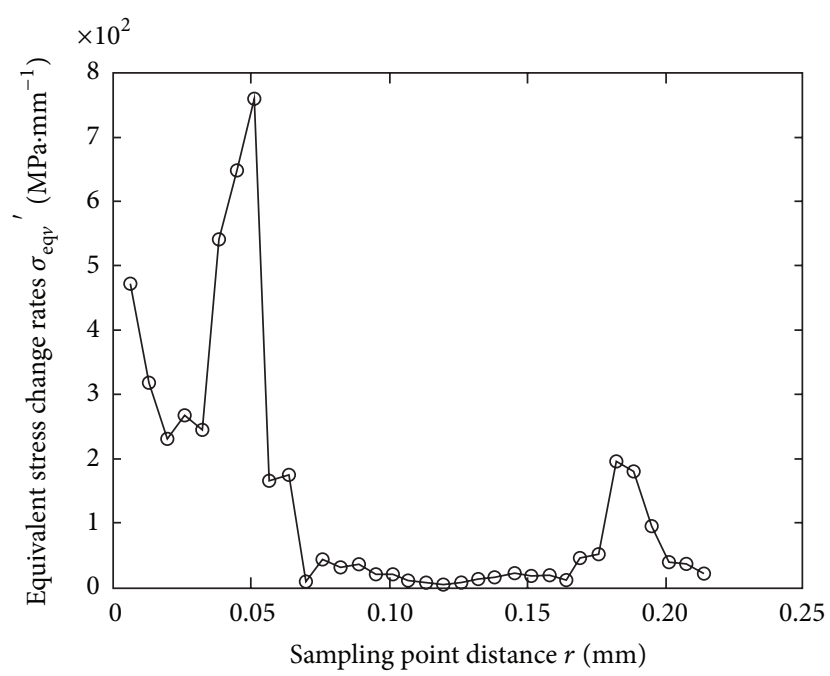

FIgURE 4: The equivalent stress change rate of the selected points.

stress point. In order to further investigate the factual fracture location, the equivalent stress change rate $\sigma_{\text {eqv }}^{\prime}$ of V-shaped notch is presented in this paper and is calculated by

$$
\sigma_{\text {eqv }}^{i}=\frac{d \sigma_{\text {eqv }}}{d r}=\frac{\left|\Delta \sigma_{\text {eqv }}\right|}{|\Delta r|}=\frac{\left|\sigma_{\text {eqv }}^{i+1}-\sigma_{\text {eqv }}^{i}\right|}{\left|r^{i+1}-r^{i}\right|}, \quad i=1, \ldots, n,
$$

where $\sigma_{\text {eqv }}^{i}$ is the equivalent stress at point $i$ on the $\mathrm{V}$-shaped notch surface and $r_{i}$ is the length between point $i$ and base point (a point very close to point $i$ ).

The results of equivalent stress change rates of the selected points at two sides of the surface near $\mathrm{V}$-shaped notch bottom are shown in Figure 4. As shown in Figure 4, the equivalent stress change rate at the transitional region between V-shaped notch bottom corner and the left side of straight surface of $\mathrm{V}$-shaped notch is maximal. Therefore, the crack initiation location is thought as the place at the maximal equivalent stress change rate on V-shaped notch surface.
2.2. Experimental Research and Analysis. The fracture geometrical forms of V-shaped notch bottom for the bars with LY8, 20 steel, and 45 steel under the action of low stress are shown in Figure 5. As shown in Figure 5, the obvious corners at V-shaped notch bottom are not found in all of the above material. The maximum corner appears in the right side of $\mathrm{V}$-shaped notch bottom for 45 steel bars, about $0.05 \mathrm{~mm}$. The corner for 20 steel bars is about $0.03 \mathrm{~mm}$. The experimental results show that these corners are the residual plastic deformations caused by the stress concentration of V-shaped notch and are not the corners produced by turning. However, the fracture surfaces of the left side of V-shaped notch bottom have obvious transitional corners, as shown in Figure 5. It is also found that the crack initiation location does not occur in center position of $\mathrm{V}$-shaped notch bottom but leans to the side of clamping the metal bar, namely, the left side of V-shaped notch bottom, and the crack initiation location mostly occurs in the transitional region between V-shaped notch bottom corner and the left side of straight surface of V-shaped notch. Because of the influences of the cutting tool manufacturing, $\mathrm{V}$-shaped notch parameters and material properties, and other factors, the region between $\mathrm{V}$-shaped notch bottom corner and two sides of straight surface of V-shaped notch is not smooth, and the local stress concentration appears easily and the microcrack occurs. Therefore, to obtain the high-quality cross-section, it should be ensured that the crack initiation point is located on the center of V-shaped notch bottom by improving the manufacturing precision of corner.

\section{The Influence of Principal Stress State on Crack Initiation Direction}

3.1. Stress Field of V-Shaped Notch Bottom. The calculating model of V-shaped notch is shown in Figure 6. $\theta$ and $r$ are the polar coordinate, and other parameters are shown in Figure 1. By means of displacement method, the stress fields of Vshaped notch tip are expressed as

$$
\begin{aligned}
\sigma_{\theta}= & \frac{S_{I}}{\sqrt{2 \pi}(r)^{1-\lambda_{1}}} \\
\times & {\left[\cos \left(1+\lambda_{1}\right) \theta\right.} \\
& \left.\quad-\frac{\left(1+\lambda_{1}\right) \sin \left(1+\lambda_{1}\right) a}{\left(1-\lambda_{1}\right) \sin \left(1-\lambda_{1}\right) a} \cos \left(1-\lambda_{1}\right) \theta\right] \\
+ & \frac{S_{I I}}{\sqrt{2 \pi}(r)^{1-\lambda_{2}}}\left[\sin \left(1+\lambda_{2}\right) \theta\right. \\
\sigma_{r}= & \left.\frac{S_{I}}{\sqrt{2 \pi}(r)^{1-\lambda_{1}}} \quad-\frac{\sin \left(1+\lambda_{2}\right) a}{\sin \left(1-\lambda_{2}\right) a} \cos \left(1-\lambda_{2}\right) \theta\right], \\
\times & {\left[-\cos \left(1+\lambda_{1}\right) \theta\right.} \\
& \left.\quad-\frac{\left(3-\lambda_{1}\right) \sin \left(1+\lambda_{1}\right) a}{\left(1-\lambda_{1}\right) \sin \left(1-\lambda_{1}\right) a} \cos \left(1-\lambda_{1}\right) \theta\right]
\end{aligned}
$$




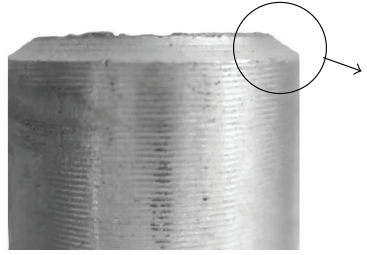

(a) Fracture surface of left side for LY8 bar

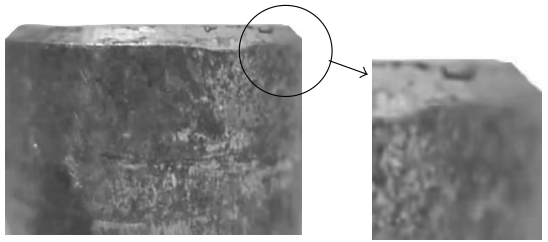

(c) Fracture surface of left side for 20 steel bar

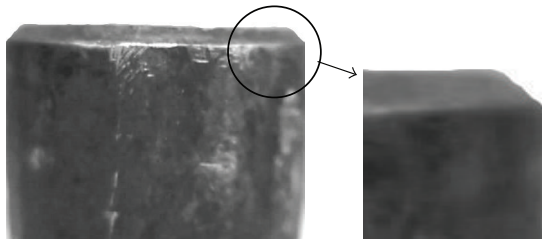

(e) Fracture surface of left side for 45 steel bar
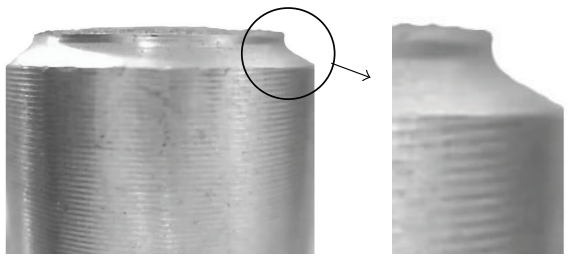

(b) Fracture surface of right side for LY8 bar

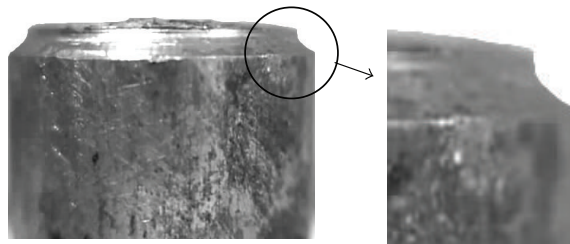

(d) Fracture surface of right side for 20 steel bar

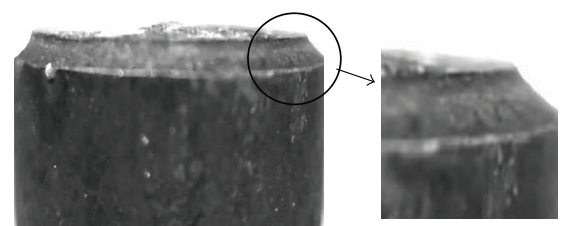

(f) Fracture surface of right side for 45 steel bar

FIGURE 5: Fracture geometrical forms of left side and right side of V-shaped notch bottom surface.

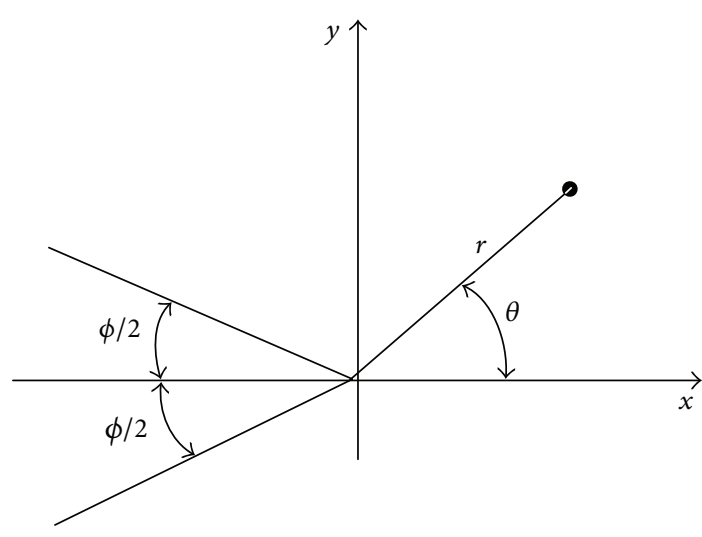

FIGURE 6: Calculating model of V-shaped notch.

$$
\begin{aligned}
& +\frac{S_{I I}}{\sqrt{2 \pi}(r)^{1-\lambda_{2}}}\left[\sin \left(1+\lambda_{2}\right) \theta\right. \\
& +\frac{\left(3-\lambda_{2}\right) \sin \left(1+\lambda_{2}\right) a}{\left(1+\lambda_{2}\right) \sin \left(1-\lambda_{2}\right) a} \\
& \left.\times \sin \left(1-\lambda_{2}\right) \theta\right] \text {, } \\
& \tau_{r \theta}=\frac{S_{I}}{\sqrt{2 \pi}(r)^{1-\lambda_{1}}} \\
& \times\left[\sin \left(1+\lambda_{1}\right) \theta-\frac{\sin \left(1+\lambda_{1}\right) a}{\sin \left(1-\lambda_{1}\right) a} \sin \left(1-\lambda_{1}\right) \theta\right]
\end{aligned}
$$

$$
\begin{aligned}
+\frac{S_{I I}}{\sqrt{2 \pi}(r)^{1-\lambda_{2}}}[ & \cos \left(1+\lambda_{2}\right) \theta \\
& -\frac{\left(1-\lambda_{2}\right) \sin \left(1+\lambda_{2}\right) a}{\left(1+\lambda_{2}\right) \sin \left(1-\lambda_{2}\right) a} \\
& \left.\times \cos \left(1-\lambda_{2}\right) \theta\right]
\end{aligned}
$$

where $S_{I}=-2 \lambda_{1} \sqrt{2 \pi} G=\left.\lim _{r \rightarrow 0} \sqrt{2 \pi}(r)^{1-\lambda_{1}} \sigma_{\theta}\right|_{\theta=0}\{1-(1+$ $\left.\left.\lambda_{1}\right) \sin \left(1+\lambda_{1}\right) a /\left(1-\lambda_{1}\right) \sin \left(1-\lambda_{1}\right) a\right\}^{-1}, S_{I I}=-2 \lambda_{2} \sqrt{2 \pi} G=$ $\left.\lim _{r \rightarrow 0} \sqrt{2 \pi}(r)^{1-\lambda_{2}} \sigma_{r \theta}\right|_{\theta=0}\left\{1-\left(1+\lambda_{2}\right) \sin \left(1+\lambda_{2}\right) a /(1-\right.$ $\left.\left.\lambda_{2}\right) \sin \left(1-\lambda_{2}\right) a\right\}^{-1}, G$ is the shear modulus of material, $\lambda_{1}$ is the first eigenvalue of $\mathrm{V}$-shaped notch, $\lambda_{2}$ is the second eigenvalue of $\mathrm{V}$-shaped notch, and $a$ is a coefficient.

By the polynomial fitting method, $\lambda_{1}$ and $\lambda_{2}$ can be expressed by

$$
\begin{aligned}
& \lambda_{1}=a_{1} \phi^{3}+a_{2} \phi^{2}+a_{3} \phi+a_{4}, \\
& \lambda_{2}=b_{1} \phi^{3}+b_{2} \phi^{2}+b_{3} \phi+b_{4},
\end{aligned}
$$

where $a_{1}=8.08 \times 10^{-8}, a_{2}=-2.38 \times 10^{-6}, a_{3}=5.88 \times 10^{-5}$, $a_{4}=0.5, b_{1}=7.24 \times 10^{-8}, b_{2}=1.23 \times 10^{-5}, b_{3}=2.86 \times 10^{-3}$, and $b_{4}=0.5$.

When the flare angle $\phi$ of $\mathrm{V}$-shaped notch is $60^{\circ}$, the first eigenvalue and the second eigenvalue are, respectively, about $\lambda_{1}=0.5122$ and $\lambda_{2}=0.7309$. Based on it, when $r \rightarrow 0$, the notch intensity factors are, respectively, about $S_{I}=-9.929 \times$

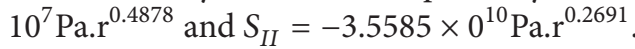




\subsection{The Calculation of Crack Initiation Angle of V-Shaped} Notch Bottom. For the metal bars, fracture mechanisms of $\mathrm{V}$-shaped notch bottom material are different under different stress states [5]. The initial defect of material is the main place of initial crack formation. The stress of the initial crack zone is generally the principal stress. Brittle fracture occurs in the action of the maximum normal stress, and the cross-section is perpendicular to the maximum principal stress. However, the ductile fracture is caused by the maximum shear stress, and the shearing fracture of material occurs along an angle of $45^{\circ}$ to the maximum principal stress. Based on the above analysis, the principal stress criterion of the crack initiation direction is proposed and the corresponding crack initiation angle is described by

$$
\begin{aligned}
& \theta_{c}=\left.\theta\right|_{\sigma_{r \theta}=0} \quad \text { Brittle material, } \\
& \theta_{c}=\left.\theta\right|_{\sigma_{r \theta}=0} \pm 45^{\circ} \quad \text { Ductile material. }
\end{aligned}
$$

For the ductile fracture, when $\sigma_{\theta}>\sigma_{r}$, (4) takes the negative value or takes positive value. Based on fracture mechanics, the stress intensity factor for type I of crack plays a leading role in determining the crack initiation direction of V-shaped notch tip [6]. For calculating the crack initiation angle, suppose that the shear stress $\tau_{r \theta}$ of $\mathrm{V}$-shaped notch tip is zero. Because $\sqrt{2 \pi} r^{1-\lambda_{1}} \neq 0$ and $\sqrt{2 \pi} r^{1-\lambda_{2}} \neq 0$, according to (2), the expression including the crack initiation angle $\theta$ is denoted as

$$
\begin{aligned}
\frac{S_{I}}{S_{I I}}=-r^{\lambda_{2}-\lambda_{1}}( & \left(\cos \left(1+\lambda_{2}\right) \theta\right. \\
& \left.-\frac{\left(1-\lambda_{2}\right) \sin \left(1+\lambda_{2}\right) a}{\left(1+\lambda_{2}\right) \sin \left(1-\lambda_{2}\right) a} \cos \left(1-\lambda_{2}\right) \theta\right) \\
& \times\left(\sin \left(1+\lambda_{1}\right) \theta\right. \\
& \left.\left.\quad-\frac{\sin \left(1+\lambda_{1}\right) a}{\sin \left(1-\lambda_{1}\right) a} \sin \left(1-\lambda_{1}\right) \theta\right)^{-1}\right) .
\end{aligned}
$$

3.3. The Theoretical Calculation and the Experimental Result of Crack Initiation Direction of V-Shaped Notch Bottom for LY8 Bar. A large number of experimental results indicate that the initial crack size is generally between $0.05 \mathrm{~mm}$ and $0.5 \mathrm{~mm}$ [7]. The size of initial crack is determined as $r=0.3 \mathrm{~mm}$. By means of (5), the crack initiation angles of four points in terms of equidistant distribution near the $\mathrm{V}$-shaped notch tip have been obtained. By the displacement extrapolation method, the crack initiation angle for LY8 bar is about $55.2^{\circ}$ when $r \rightarrow 0$. The calculation results show that $\sigma_{\theta}>\sigma_{r}$, and the crack initiation angle of $\mathrm{V}$-shaped notch tip for the ductile material is about $\theta_{c}=55.2^{\circ}-45^{\circ}=10.2^{\circ}$.

In order to observe the factual crack initiation angle, the microscopic measurement system is built. The experimental system mainly consists of microscope, electron eyepiece, objective microscale, light source, and computer. When measuring, the test sheet metal is placed on stage, and then it

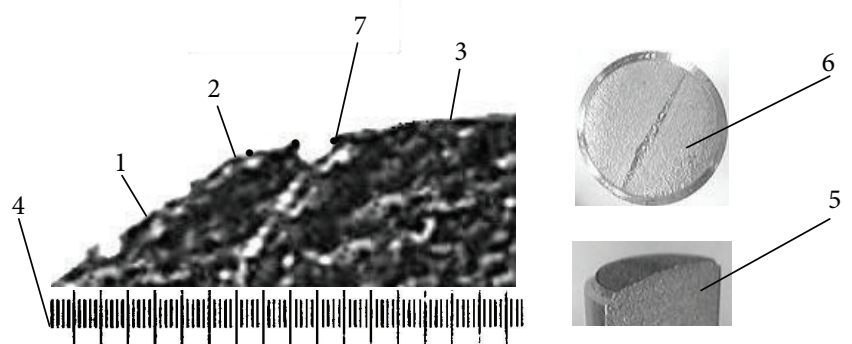

(1) Notch surface

(2) V-shaped notch tip zone

(3) Fracture surface

(4) Objective microscale

(5) Test sheet metal

(6) Whole fracture surface of bar

(7) Analytical point

FigURE 7: The microstructure near V-shape notch tip surface.

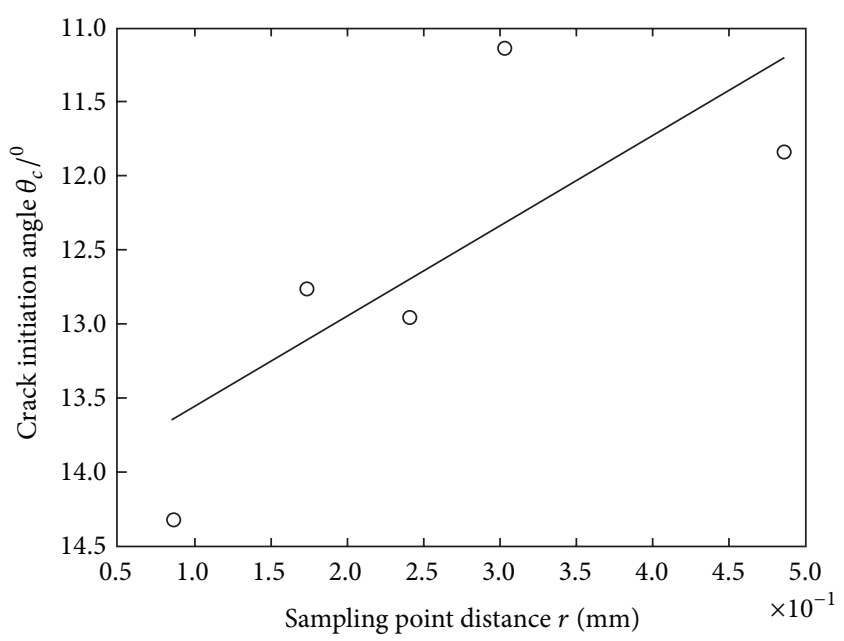

Figure 8: Displacement extrapolation curve for LY8 bar.

adjusts the light source; the clear microscopic image of $\mathrm{V}$ shaped notch tip for LY8 bar can be obtained as shown in Figure 7. The tip point of $\mathrm{V}$-shaped notch is regarded as the starting point and other 5 points of fracture surface are regarded as analytical points. Then the slopes between these analytical points and the starting point are calculated, respectively. Finally, by means of the displacement extrapolation method, the displacement extrapolation curve is shown in Figure 8, and the crack initiation angle of $\mathrm{V}$-shaped notch tip for LY8 bar is about $\theta_{c}=11.3^{\circ}$.

The calculation result and the experimental result have a difference of about $9.7 \%$. Main reasons are analyzed as follows: (1) the influence of measurement error, machining error, and other factors and (2) V-shaped notch tip being regarded as the sharp notch. However, the factual V-shaped notch bottom has the radius of corner. The sharper the $\mathrm{V}$ shaped notch bottom, the smaller the crack initiation angle. As a whole, the theoretical results have a good agreement with 
one of the measurement results, which further proves that the calculation formula of crack initiation angle proposed in the paper is correct.

\section{The Calculation of Crack Initiation Life of V-Shaped Notch Bottom}

4.1. The Formula of Crack Initiation Life of V-Shaped Notch Tip. The crack initiation life of V-shaped notch tip for the metal bar is defined as the stress-cycle number when the crack initiation length is about $0.25-0.3 \mathrm{~mm}$ [7]. There are many estimation methods of crack initiation time of $\mathrm{V}$ shaped notch bottom. Buch et al. investigated the fatigue crack initiation time under the constant amplitude loading by the local stress-strain method and thought that the estimated accuracy mainly depended on the fatigue notch factor [8]. Forman determined the crack initiation time with notched specimen and regarded the crack initiation time as a function of stress intensity factor [9]. Grover et al. proposed a simple calculation formula of crack initiation time, which included the material parameters [10]; however, the formula did not give the determining method of the stress concentration coefficient and the tensile properties of material. Therefore, for the metal bar with $\mathrm{V}$-shaped notch, a specific formula of crack initial life is proposed in this paper.

The size of initial crack of $\mathrm{V}$-shaped notch is still determined as $r=0.3 \mathrm{~mm}$. Because the stress or strain concentration firstly occurs in the tip of V-shaped notch, the microcrack is formed in the $\mathrm{V}$-shaped notch bottom. When the V-shaped notch tip of metal bar is subjected to the cyclic nominal stress range $\Delta \sigma$, the material element of $\mathrm{V}$-shaped notch bottom will be subjected to the cyclic strain range $\Delta \varepsilon$ because of stress concentration effect. Therefore, the fatigue fracture of material element of V-shaped notch bottom under the action of $\Delta \varepsilon$ is regarded as the formation of fatigue microcrack of $\mathrm{V}$-shaped notch bottom under the action of $\Delta \sigma$. The above assumption has been clearly stated in [11].

According to the above theory, if the material element of $\mathrm{V}$-shaped notch bottom is equivalent to the smooth bar, the fatigue life $N_{f}$ for the smooth bar under the action of $\Delta \varepsilon$ is regarded as the crack initiation time $N_{i}$ under the action of nominal stress range $\Delta \sigma$. The strain range fatigue life for the smooth bar is expressed as [12]

$$
N_{f}=\varepsilon_{f}^{2}\left(\Delta \varepsilon-\Delta \varepsilon_{c}\right)^{2},
$$

where $\Delta \varepsilon$ is the local strain range of $\mathrm{V}$-shaped notch bottom, $\varepsilon_{f}$ is the fracture ductility of material, and $\Delta \varepsilon_{c}$ is the theoretical strain fatigue limit, and its expression is $\Delta \varepsilon_{c} \approx$ $\sigma_{b} / E-\varepsilon_{f} / 10^{3.5}$.

The relationship between $\Delta \varepsilon, \Delta \sigma$ and stress ratio $R$ is expressed as follows:

$$
\Delta \varepsilon=2\left[\frac{1}{E K} \Delta \sigma_{\mathrm{egv}}^{2}\right]^{1 /(n+1)},
$$

where $E$ is the elastic modulus of material, $K$ is the strength factor, expressed by $K=\sigma_{f} / \varepsilon_{f}^{n}$, and $\Delta \sigma_{\text {egv }}$ is the equivalent stress amplitude, expressed by $\Delta \sigma_{\text {egv }}=\sqrt{1 / 2(1-R)} k_{\phi} \Delta \sigma$, where $k_{\phi}$ is the stress concentration factor of the notch, and its expression is $k_{\phi}=1+(2 \sqrt{d / s} f(d / b)-1) \times[1-(\phi /$ $\left.180)^{1+2.4 \sqrt{s / d}}\right]$, where $f(d / b)=(3 / 8) \sqrt{(1-d / b)}[1+(1 / 2)(1-$ $d / b)+(3 / 8)(1-d / b)^{2}+(5 / 16)(1-d / b)^{3}+(35 / 128)(1-$ $\left.d / b)^{4}+0.537(1-d / b)^{5}\right]$.

By substituting (7) into (6) and replacing $N_{f}$ by $N_{i}$, the whole formula of crack initial life of $\mathrm{V}$-shaped notch tip is expressed as

$$
N_{i}=C_{i}\left[\Delta \sigma_{\mathrm{egv}}^{2 /(1+n)}-\left(\Delta \sigma_{\mathrm{egv}}\right)_{\mathrm{th}}^{2 /(1+n)}\right]^{-2}
$$

where $n$ is the strain hardening index, $C_{i}$ is the resistance coefficient of material, expressed by $C_{i}=(1 / 4)\left(\sqrt{E \sigma_{f} \varepsilon_{f}}\right)^{4 /(1+n)}$, $\sigma_{f}$ is the tensile fracture strength, expressed by $\sigma_{f}=\sigma_{b}(1+$ $\left.\psi_{k}\right)$, and $\left(\Delta \sigma_{\text {egv }}\right)_{\text {th }}$ is the crack initiation threshold, expressed by $\left(\Delta \sigma_{\text {egv }}\right)_{\text {th }}=\sqrt{E \sigma_{f} \varepsilon_{f}}\left(\Delta \varepsilon_{c} / 2 \varepsilon_{f}\right)^{(1+n) / 2}$.

Therefore, the crack initiation time of $\mathrm{V}$-shaped notch tip is related to the geometry of V-shaped notch, the cyclic loading condition, the tensile property of material, and the crack initiation threshold. For metal bars with different material, the initial force acted on the bar, and the geometric parameters of $\mathrm{V}$-shaped notch should be determined reasonably to reduce the crack initiation life.

4.2. The Experimental Results of Crack Initiation Life. Taking 45 steel bars as a sample to calculate the crack initiation life of V-shaped notch in the low stress cropping, the chemical compositions and mechanical parameters of 45 steel bar material are shown in Tables 1 and 2, respectively. In the existing parameters, by means of (8), the crack initiation life of $\mathrm{V}$-shaped notch is about $17.9 \mathrm{~s}$ when the initial loading force is equal to $1388 \mathrm{~N}$. The obtained crack initiation time by the local stress-stain method is about $61 \mathrm{~s}$ when the initiation crack size is $0.1 \mathrm{~mm}$. However, the factual cropping time for every segment of 45 steel bars is about $20 \mathrm{~s}$, the theoretical crack extension time is $3.6 \mathrm{~s}$, and thus the crack initiation time is about $16.4 \mathrm{~s}$. When the initial loading force is equal to $1757 \mathrm{~N}$, the crack initiation time of V-shaped notch is about $6.8 \mathrm{~s}$ by means of (8). The factual cropping time for every segment of bar is about $8 \mathrm{~s}$, the theoretical crack expansion time is $1.6 \mathrm{~s}$, and thus the crack initiation time is about $6.4 \mathrm{~s}$. Therefore, the calculation formula of the crack initiation life presented in this paper is accurate. In addition, it also showed that the crack initiation life of $\mathrm{V}$-shaped notch tip is more than $80 \%$ of the total cropping time for every segment of metal bar. A series of cropping experiments for 20 steel bars have been performed, and the obtained conclusion is the same with the one for 45 steel bars.

\section{Conclusions}

The crack initiation behavior of V-shaped notch tip is studied. Much experimental evidence obtained in bar cropping supports the research results obtained in this paper. The work is instructive for further investigation on the new type of low stress precision cropping. By means of combining theory 
TABLE 1: Chemical compositions of 45 steel bar material.

\begin{tabular}{lc}
\hline Chemical compositions & Values \\
\hline $\mathrm{C} / \%$ & $0.37 \sim 0.52$ \\
$\mathrm{Mn} / \%$ & $0.51 \sim 0.81$ \\
$\mathrm{Si} / \%$ & $0.28 \sim 0.48$ \\
$\mathrm{~S} / \%$ & $0.01 \sim 0.029$ \\
$\mathrm{P} / \%$ & $0.017 \sim 0.044$ \\
\hline
\end{tabular}

TABle 2: Parameters of 45 steel bars.

\begin{tabular}{lc}
\hline Mechanical parameters & Values \\
\hline Diameter $2 b / \mathrm{mm}$ & 15 \\
Notch depth $d / \mathrm{mm}$ & 1.5 \\
Corner radius $s / \mathrm{mm}$ & 0.2 \\
Flare angle $\phi /^{\circ}$ & 60 \\
Fracture toughness $K_{\mathrm{IC}} / \mathrm{MPa} \cdot \mathrm{m}^{0.5}$ & 102.78 \\
Crack extension threshold $\Delta K_{\mathrm{th}} / \mathrm{MPa} \cdot \mathrm{m}^{0.5}$ & 8 \\
Tensile ultimate strength $\sigma_{b} / \mathrm{MPa}$ & 639 \\
Yield limit $\sigma_{s} / \mathrm{MPa}$ & 377 \\
Contraction of cross section area $\psi_{k}$ & 0.4 \\
Fracture ductility $\varepsilon_{f}$ & 0.465 \\
Strain hardening coefficient $n$ & 0.179 \\
Elastic modulus $E / \mathrm{N} \cdot \mathrm{m}^{-2}$ & $210 \times 10^{9}$ \\
Extensibility & $22.02 \%$ \\
\hline
\end{tabular}

with experiment, the following summarizes in detail the contributions made in the present study.

(1) In course of the low stress precision cropping, the fracture behavior of the metal bar with annular Vshaped notch includes the crack initiation at Vshaped notch bottom, the crack extension behavior, and the instant fracture process. The crack initiation is a key process and directly affects the cropping time and the cross-section quality. The results show that the crack initiation time of V-shaped notch tip accounts for about $80 \%$ of the total cropping time of every segment of metal bar.

(2) The crack initiation location mostly occurs in the transitional region between $\mathrm{V}$-shaped notch bottom corner and two sides of straight surface of V-shaped notch. The smoothness and continuity of corner of $\mathrm{V}$-shaped notch bottom are important factors, which directly affect the crack initiation location. Both numerical analyses and experimental results show that the crack initiation location is the place at the maximal equivalent stress change rate on $\mathrm{V}$-shaped notch surface.

(3) The criterion of crack initiation direction is given. According to the metal bars with different material, the formula of crack initiation angle is proposed in this paper, and the cropping experimental results for LY8 bars show that the formula of crack initiation angle is feasible. The sharper the V-shaped notch bottom, the smaller the crack initiation angle.
(4) The formula of crack initiation life of V-shaped notch tip is obtained and it includes the geometry of $\mathrm{V}$-shaped notch, the cyclic loading conditions, the tensile performance parameters of material, and the crack initiation threshold. The experimental results show that the formula of the crack initiation life presented in this paper is accurate and can be applied easily.

\section{Conflict of Interests}

The authors declare that there is no conflict of interests regarding the publication of this paper.

\section{Acknowledgments}

The authors wish to express gratitude to the National Natural Science Foundation of China (approval no. 51105382) and Shandong Natural Science Foundation of China (approval no. ZR2011EL039), which have supported this work.

\section{References}

[1] L. J. Zhang, S. D. Zhao, J. Lei, and W. Liu, "Investigation on the bar clamping position of a new type of precision cropping system with variable frequency vibration," International Journal of Machine Tools and Manufacture, vol. 47, no. 7-8, pp. 1125-1131, 2007.

[2] Y. Tang, S. D. Zhao, and J. Lin, "Experimental investigation of the effect of the circumferential loading of a rotary striking action cropping system," Proceedings of the Institution of Mechanical Engineers, Part B: Journal of Engineering Manufacture, vol. 224, no. 7, pp. 1095-1101, 2010.

[3] L. J. Zhang, S. D. Zhao, C. J. Hua, C. F. Guo, and J. Lei, "Investigation on a new type of low-stress cropping system with variable frequency vibration," International Journal of Advanced Manufacturing Technology, vol. 36, no. 3-4, pp. 288-295, 2008.

[4] X. L. Zhang, Investigation in the mechanism of a low stress precision bar cropping system with variant frequency vibration method, [Master thesis], Xi'an Jiao Tong University, Xi'an, China, 2005.

[5] H. Min, Y. Yue, and W. Ling, Material Mechanics, Xi'an Jiaotong University, Xi'an, China, 1999.

[6] Z. N. Li and Q. Z. He, Engineering Fracture Mechanics, Beihang University press, Beijing, China, 1993.

[7] X. L. Zheng, The Quantitative Theory of Metal Fatigue, Northwestern Polytechnical University Press, Xi'an, China, 1994.

[8] A. Buch, T. Seeger, and M. Vormwald, "Improvement of fatigue life prediction accuracy for various realistic loading spectra by use of correction factors," International Journal of Fatigue, vol. 8, no. 4, pp. 175-185, 1986.

[9] R. G. Forman, "Study of fatigue crack initiation from flaws using fracture mechanics theory," Engineering Fracture Mechanics, vol. 4, no. 2, pp. 333-345, 1972.

[10] H. J. Grover, A. Gordon, and L. R. Jackson, Fatigue of Metals and Structures, US Government Printing Office, Washington, DC, USA, 1954 . 
[11] L. F. Coffin, "Fatigue in machines and structures-power generation," in Proceedings of the Materials Science Seminar, Fatigue and Microstructures, pp. 4-7, American Society for Metals, St. Louis, Mo, USA, 1978.

[12] X. L. Zheng and G. Z. Zhang, "Study of strain fatigue formula," Journal of Northwestern Polytechnical University, vol. 2, no. 2, pp. 223-229, 1984. 

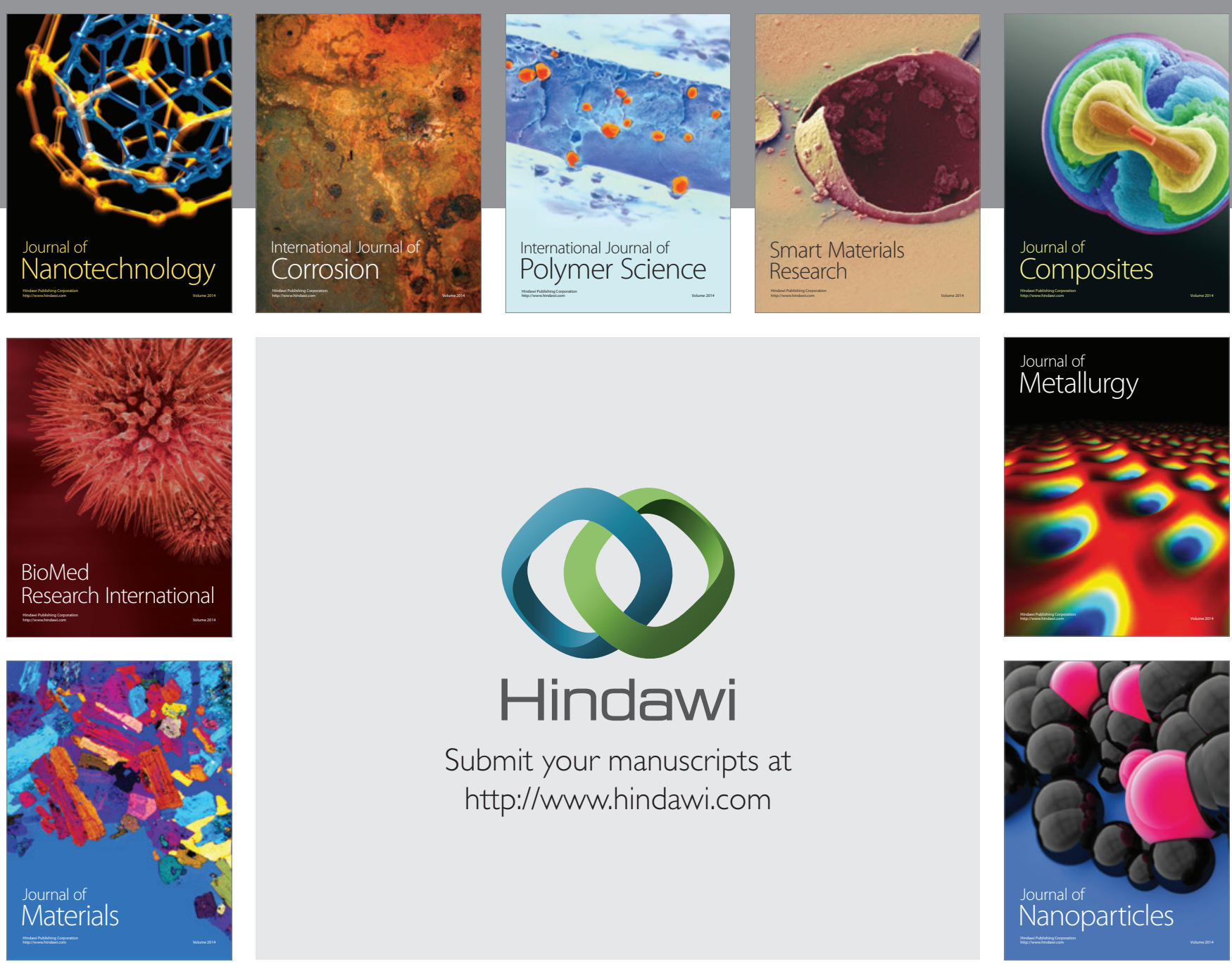

Submit your manuscripts at http://www.hindawi.com
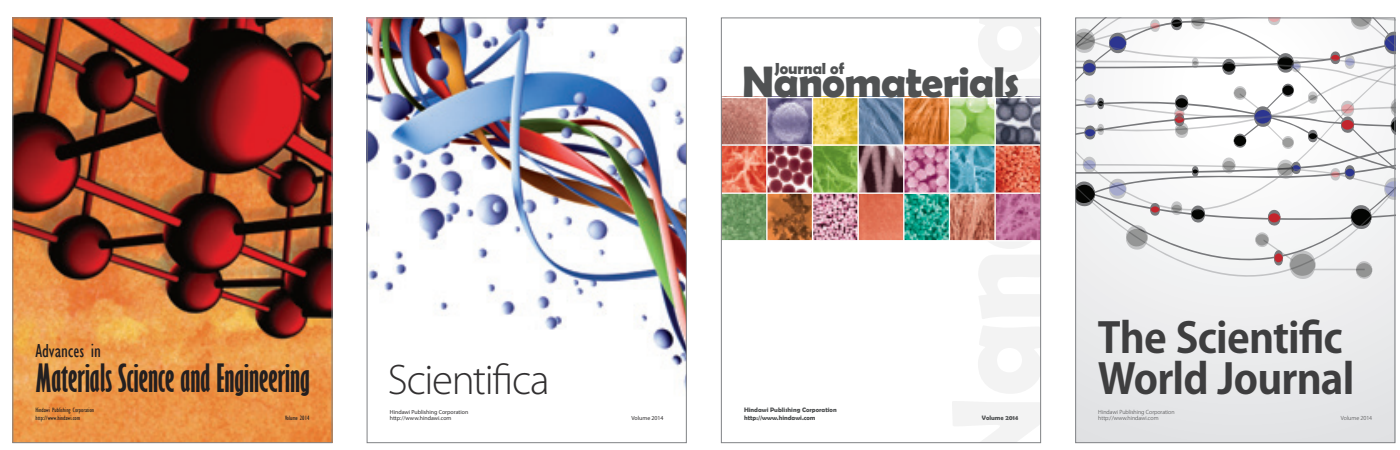

\section{The Scientific World Journal}
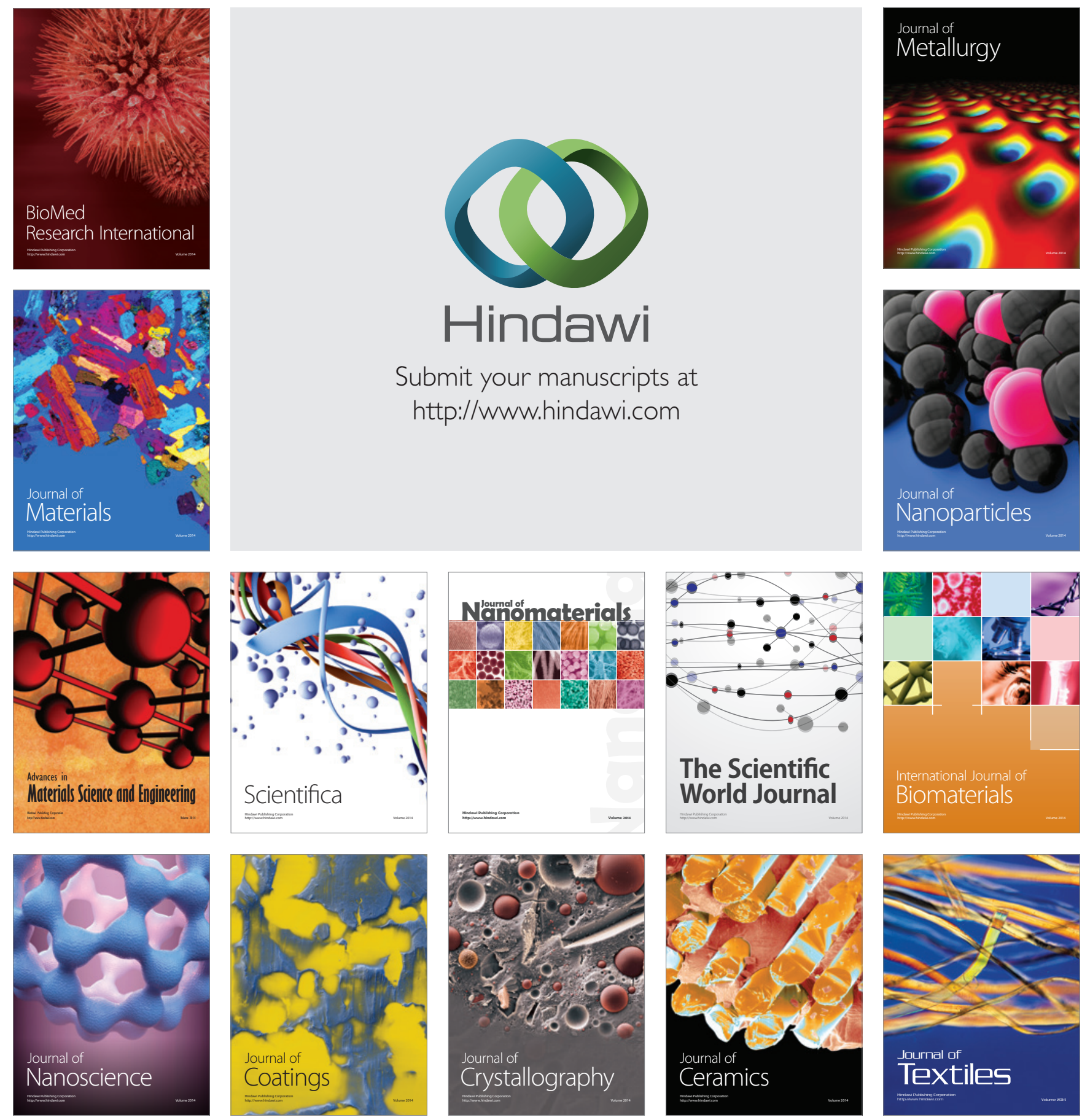